\section{(2) OPEN ACCESS}

\title{
Guideline-directed therapy at discharge in patients with heart failure and atrial fibrillation
}

\author{
Min-Soo Ahn (D) ,' Byung Su Yoo (D) ,' Junghan Yoon (D) ,' Seung-Hwan Lee (D) ,' \\ Jang Young Kim (D) ,' Sung Gyun Ahn (D) ,' Young Jin Youn (D) ,' Jun-Won Lee (D) ,' \\ Jung-Woo Son (D) ,' Hye Sim Kim (D) ,' Dae Ryong Kang (D) , ${ }^{2}$ Hyun-Jai Cho (D) , \\ Hae-Young Lee (D), ${ }^{4}$ Eun Seok Jeon (D) , ${ }^{5}$ Seok-Min Kang (D) , ${ }^{6}$ Dong-Ju Choi (i) , \\ Myeong-Chan Cho (D) ${ }^{8}$
}

\begin{abstract}
- Additional material is published online only. To view, please visit the journal online (http://dx.doi.org/10. 1136heartjnl-2019-315240)
\end{abstract}

For numbered affiliations see end of article.

\section{Correspondence to} Professor Byung Su Yoo, Department of Internal Medicine, Yonsei University, Wonju College of Medicine, Wonju 26426, Republic of Korea; yubs@yonsei.ac.kr

This abstract was presented as a poster presentation at EHRA 2019.

Received 13 April 2019 Revised 8 July 2019 Accepted 24 July 2019 Published Online First 6 September 2019

\section{Check for updates}

(C) Author(s) (or their employer(s)) 2020. Re-use permitted under CC BY-NC. No commercial re-use. See rights and permissions. Published by BMJ.

To cite: Ahn M-S, Yoo BS, Yoon J, et al. Heart

2020;106:292-298.

\begin{abstract}
Objectives This study evaluated the relationship between guideline adherence for recommended therapy on discharge and relevant 60-day and 1-year clinical outcomes in patients with acute heart failure (HF) with reduced ejection fraction and atrial fibrillation (AF). Methods Of 5625 acute patients with $\mathrm{HF}$ in the Korean Acute Heart Failure registry, 986 patients with HF and documented AF were analysed. Guideline adherence scores were calculated for the prescription of ACE inhibitors, angiotensin receptor blockers, $\beta$-blockers, mineralocorticoid receptor antagonists and anticoagulants.
\end{abstract}

Results In patients with $\mathrm{HF}$ with $\mathrm{AF}$, there was a significant trend of reduced 60-day and 1-year mortality rates and the composite end point with guideline adherence. According to the Cox proportion hazard model, poor adherence was associated with a significantly higher risk of 60-day mortality (HR 4.75; $95 \% \mathrm{Cl} 1.77$ to 12.74 ) and the composite end point (HR 2.36; $95 \% \mathrm{Cl} 1.33$ to 4.18 ) compared with good adherence. Furthermore, poor adherence was associated with a significantly higher risk of 1-year mortality compared with moderate (HR 1.64; $95 \% \mathrm{Cl} 1.15$ to 2.33) and good adherence (HR $2.34 ; 95 \% \mathrm{Cl} 1.39$ to 3.97 ) and with a higher risk of the 1-year composite end point compared with good adherence (HR 1.58; 95\% Cl 1.07 to 2.33).

Conclusion Better adherence to guidelines was associated with better 60-day and 1-year prognoses in patients with $\mathrm{HF}$ with AF.

\section{INTRODUCTION}

Atrial fibrillation (AF) is common in patients with heart failure (HF) because the pathophysiology and risk factors for $\mathrm{HF}$ and $\mathrm{AF}$ are closely associated. ${ }^{1}$ Regardless of which comes first, patients with concomitant $\mathrm{HF}$ and AF have significantly poor prognoses. $^{2}$ Acute HF (AHF) is a life-threatening medical condition with a high inpatient mortality (typically $>4 \%$ ); however, most cases of morbidity and mortality occur after the index hospital discharge. ${ }^{3}$ Therefore, comprehensive strategies should focus on factors during hospitalisation and also on those during the early recovery period soon after discharge to target stressors that contribute to patient vulnerability. HF guidelines recommend that evidence-based oral disease-modifying HF therapy should be started or continued on admission with $\mathrm{AFH}$, except in the presence of a contraindication. $^{4-6}$ The evidence-based benefits of guidelinedirected therapy for HF have been documented, including reduced ejection fraction (HFrEF) and normal sinus rhythm, but there are limited and conflicting data regarding the prognostic implications of guideline-directed therapy for patients with acute HFrEF with AF. Thus, this study assessed the relationship between guideline adherence on discharge and relevant 60-day and 1-year clinical outcomes in patients with acute HFrEF with AF.

\section{METHODS}

\section{Study population}

This study used the registry of the Korean Acute Heart Failure (KorAHF) study, a multicentre prospective cohort study that enrolled 5,625 consecutive patients who were hospitalised for AHF at 10 tertiary university hospitals between March 2011 and February 2014. The patients were scheduled for follow-up until the end of 2018. The registry included patients with signs or symptoms of HF who met at least one of the following criteria: (1) lung congestion or (2) objective findings of left ventricular (LV) systolic dysfunction or structural heart disease. Lung congestion was defined as 'congestion' on chest radiography or as rales on physical examination. The study protocol was approved by the ethicscommittee of each hospital. Detailed information on the study design and the results of the KorAHF registry has been provided previously. ${ }^{7}$ From this population, we selected patients with $\mathrm{HFrEF}$ and AF who had survived hospitalisation with 1 year of follow-up data.

\section{Modified guideline adherence score}

To assess adherence to guidelines, a modified guideline adherence score was calculated. This score was adopted and modified from the QUality of Adherence to guideline recommendations for LIFe-saving treatment in heart failure surveY (QUALIFY) international survey. ${ }^{8}$ A global guideline adherence score in QUALIFY was constructed on the basis of physicians' adherence to guidelines regarding prescription of the following classes of medications: ACE inhibitors (ACEIs), angiotensin receptor blockers (ARBs), 
$\beta$-blockers, mineralocorticoid receptor antagonists (MRAs) and ivabradine, which are recommended by the American College of Cardiology (ACC)/American Heart Association (AHA)/European Society of Cardiology (ESC) guidelines for patients with HF with normal sinus rhythm, unless contraindicated or not tolerated. ${ }^{468}$ However, ivabradine was excluded from this study because it is only indicated for patients with a normal sinus rhythm. Alternatively, anticoagulation therapy was included as guideline-recommended therapy because patients with HF and AF should generally receive anticoagulation therapy to balance the benefit and risk of bleeding (using $\mathrm{CHA}_{2} \mathrm{DS}_{2}$-VASc and HAS-BLED scores). ${ }^{9}{ }^{10}$ In addition to the reduction of stroke and distal embolisation, anticoagulants markedly reduce the risk of death; the salutary effect of warfarin may be multifactorial. ${ }^{11}$ The adherence score was the ratio of treatment actually prescribed to the treatment that should have theoretically been prescribed. The score was calculated for each patient by summing the following points: 0 for non-prescription in the absence of specified contraindications and 1 point each for the use of ACEIs or ARBs, $\beta$-blockers, MRAs and oral anticoagulation (if indicated). Only guideline-recommended $\beta$-blockers, including bisoprolol, carvedilol, sustained-release metoprolol succinate and nebivolol were scored as adherence to guidelines. Nonadministration of recommended drugs because of specific contraindications or intolerance was scored as adherence to guidelines. ${ }^{8}$ Moreover, non-administration of anticoagulation for a $\mathrm{CHA}_{2} \mathrm{DS}_{2}$ VASc score $<2$ was scored as adherence. The score ranged from 0 to 1 , and three levels of adherence were defined: good (use of all indicated medications; score $=1$ ), moderate (use of more than half of the indicated medications; $0.5 \leq$ score $<1$ ) and poor (use of $<50 \%$ of the indicated medications; score $<0.5$ ) adherence. ${ }^{812}$

\section{Clinical outcomes}

Clinical outcomes after discharge in the KorAHF registry included all-cause death, death from HF aggravation and rehospitalisation for HF aggravation. The attending physician collected follow-up data from the patients and stored them in a web-based case report form. The outcome data for subjects who were not followed up were ascertained by a telephone interview. In addition, the outcome data for patients who were lost to follow-up were ascertained from the National Death Records. All clinical events were monitored and verified by a Clinical Event Committee, comprising independent experts on HF who did not participate in patient enrolment for the study. ${ }^{7}$ In this study, the outcomes were defined as 60-day and 1-year all-cause mortality, rehospitalisation because of aggravated $\mathrm{HF}$ and a composite end point of mortality and HF rehospitalisation.

\section{Statistical analysis}

Continuous variables are reported as mean \pm SD and were compared using analysis of variance. Categorical variables are presented as frequencies and percentages and were compared using the $\chi^{2}$ test. Individual and composite clinical outcomes were analysed on the basis of time to the first event. Kaplan-Meier survival curves were plotted, and the groups were compared using the logrank test. To assess the relationship between outcomes and guide adherence, Cox proportional hazards regression models were used. The models were adjusted for prespecified baseline covariates, including sex, age, body mass index, history of hypertension, history of diabetes mellitus, history of ischaemic heart disease, history of chronic obstructive pulmonary disease, type of HF (de novo vs acute decompensated HF), New York Heart Association functional class, systolic blood pressure, heart rate, creatinine at admission and left ventricular ejection fraction (LVEF). Competing risk analysis, using the subdistribution hazard model proposed by

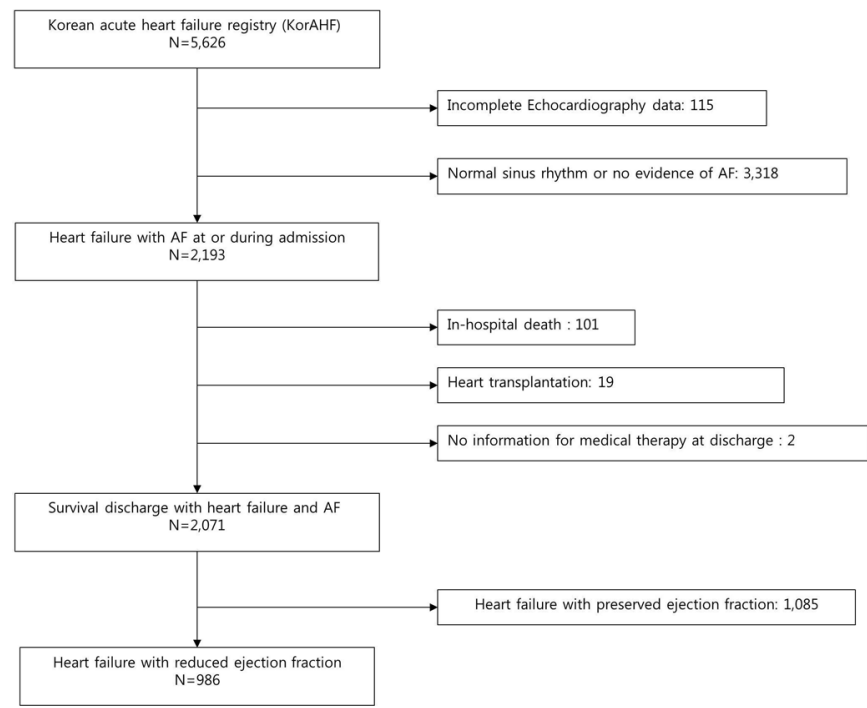

Figure 1 Flow diagram of patients included in the study from theKorAHF. AF, atrial fibrillation.

Fine and Gray, was used to examine associations of the guideline adherence with HF rehospitalisation by accounting for competing risks of death. ${ }^{13}$ This method adjusts for the potential preclusion of the occurrence of the event of interest by another event occurring earlier, the competing event. In all cases, a $\mathrm{p}$ value $\leq 0.05$ was considered statistically significant. Statistical analyses were performed using SPSS V.23.0 (IBM, USA) and SAS V.9.4 (SAS, Cary, North Carolina, USA).

\section{RESULTS}

\section{Baseline characteristics and medication at discharge of the study population}

Of the 5625 patients in the KorAHF registry, 4640 were excluded because of a normal sinus rhythm, HF with preserved ejection fraction, in-hospital death, need for transplantation, incomplete echocardiographic or electrocardiographic data or incomplete data on discharge medication (figure 1). Finally, 986 patients with HF with AF were included in this study. The modified guideline adherence score was good in $21 \%$, moderate in $64.1 \%$ and poor in $14.9 \%$ of the patients. As expected, the patients had unfavourable clinical characteristics with a lower guideline adherence score. There was a significant trend towards decreased guideline adherence with increasing age. The characteristics of poor guideline adherence were as follows: lower body mass index, high proportion of chronic kidney disease, lower levels of sodium and haemoglobin and lower proportion of de novo HF. The $\mathrm{CHA}_{2} \mathrm{DS}_{2}-\mathrm{VASc}$ score was inversely correlated with guideline adherence $(3.9 \pm 1.7$ vs $3.7 \pm 1.7$ vs $3.2 \pm 1.6, \mathrm{p}<0.001)$. The morphofunctional parameters measured by echocardiography were similar (table 1).

In the poor adherence group, the adherence rates for guidelinedirected therapies were as follows: $20.4 \%$ for use of ACEIs or ARBs, $16.3 \%$ for $\beta$-blockers, $12.2 \%$ for MRAs and $25.9 \%$ for warfarin. The calculated equivalent doses of each medication did not significantly differ according to adherence. The prescription rates of other medications, including loop diuretics and digitalis, were also significantly lower in the poor adherence group (table 2).

\section{Clinical outcomes}

In total, $200(20.3 \%)$ patients died and 200 (20.3\%) patients were rehospitalised during the 1-year follow-up period. The incidences of 60-day mortality and the composite end point increased with a decrease in guideline adherence $(\mathrm{p}<0.001)$. These events most 
Table 1 Baseline characteristics of patients according to the modified guideline adherence score

\begin{tabular}{|c|c|c|c|c|}
\hline & Poor $(n=147)$ & Moderate $(n=632)$ & Good $(n=207)$ & $P$ value \\
\hline \multicolumn{5}{|c|}{ Demographic characteristics at admission } \\
\hline Age, years & $72.8 \pm 13.1$ & $69.2 \pm 12.3$ & $64.3 \pm 13.5$ & $<0.001$ \\
\hline Male, \% & 38.8 & 39.9 & 28.5 & 0.01 \\
\hline $\mathrm{BMI}, \mathrm{kg} / \mathrm{m}^{2}$ & $22.4 \pm 3.6$ & $23.1 \pm 3.7$ & $24.2 \pm 3.9$ & $<0.001$ \\
\hline $\mathrm{sBP}, \mathrm{mm} \mathrm{Hg}$ & $126.3 \pm 27.1$ & $126.3 \pm 27.2$ & $127.1 \pm 26.8$ & 0.83 \\
\hline $\mathrm{dBP}, \mathrm{mm} \mathrm{Hg}$ & $76.2 \pm 17.9$ & $79.4 \pm 18.3$ & $82.2 \pm 18.4$ & 0.01 \\
\hline Pulse rate, beats/min & $102.0 \pm 29.6$ & $99.2 \pm 29.4$ & $97.6 \pm 27.7$ & 0.36 \\
\hline De novo heart failure & $57(38.8)$ & $287(45.4)$ & $108(52.2)$ & 0.04 \\
\hline NYHA functional class & & & & 0.24 \\
\hline II, n (\%) & $17(11.6)$ & $90(14.2)$ & $22(10.6)$ & \\
\hline III, n (\%) & $57(38.8)$ & $241(38.1)$ & $96(46.4)$ & \\
\hline $\mathrm{IV}, \mathrm{n}(\%)$ & $73(49.7)$ & $301(47.6)$ & $89(43.0)$ & \\
\hline \multicolumn{5}{|l|}{ Comorbidity } \\
\hline Hypertension, n (\%) & $78(53.1)$ & $353(55.9)$ & $109(52.7)$ & 0.66 \\
\hline $\mathrm{DM}, \mathrm{n}(\%)$ & $42(28.6)$ & $197(31.2)$ & $57(27.5)$ & 0.56 \\
\hline IHD, n (\%) & $29(19.7)$ & $169(26.7)$ & $34(16.7)$ & 0.01 \\
\hline COPD, n (\%) & $22(15.0)$ & $78(12.3)$ & $16(7.7)$ & 0.09 \\
\hline CKD, n (\%) & $27(18.4)$ & $80(12.7)$ & $18(8.7)$ & 0.03 \\
\hline CVD, n (\%) & $29(19.7)$ & $100(15.8)$ & $34(16.4)$ & 0.52 \\
\hline $\mathrm{CHA}_{2} \mathrm{DS}_{2}$-VASc score & $3.9 \pm 1.7$ & $3.7 \pm 1.7$ & $3.2 \pm 1.6$ & $<0.001$ \\
\hline Aetiology of heart failure & & & & 0.01 \\
\hline IHD, n (\%) & $46(31.3)$ & $192(30.4)$ & $41(19.8)$ & \\
\hline VHD, n (\%) & $24(16.3)$ & $82(13.0)$ & $15(7.2)$ & \\
\hline Cardiomyopathy, n (\%) & $36(24.5)$ & $166(26.3)$ & $79(38.2)$ & \\
\hline \multicolumn{5}{|c|}{ ECG characteristics at admission } \\
\hline RBBB & $15(10.2)$ & $37(5.9)$ & $13(6.3)$ & 0.16 \\
\hline LBBB & $8(5.4)$ & $38(6.0)$ & $8(3.9)$ & 0.50 \\
\hline QTC & $484.3 \pm 57.5$ & $478.0 \pm 47.7$ & $484.7 \pm 42.2$ & 0.12 \\
\hline \multicolumn{5}{|c|}{ Laboratory characteristics at admission } \\
\hline $\mathrm{Na}, \mathrm{mmol} / \mathrm{L}$ & $136.7 \pm 5.3$ & $137.3 \pm 4.8$ & $138.5 \pm 3.6$ & 0.001 \\
\hline Haemoglobin, g/L & $127.2 \pm 20.8$ & $129.6 \pm 22.8$ & $136.2 \pm 23.1$ & $<0.001$ \\
\hline Creatinine, mg/dL & $1.39 \pm 0.60$ & $1.48 \pm 1.36$ & $1.37 \pm 1.18$ & 0.48 \\
\hline BNP, pg/mL & $1777.2 \pm 1850.7$ & $1342.4 \pm 1340.5$ & $1381.6 \pm 1090.3$ & 0.14 \\
\hline Troponin I, mg/mL & $2.1 \pm 8.0$ & $1.1 \pm 6.0$ & $0.3 \pm 1.2$ & 0.04 \\
\hline \multicolumn{5}{|c|}{ Echocardiographic characteristics } \\
\hline LVEDV, mL & $159.0 \pm 80.4$ & $160.6 \pm 64.4$ & $171.8 \pm 74.1$ & 0.20 \\
\hline LVESV, mL & $114.0 \pm 67.3$ & $115.6 \pm 50.7$ & $127.1 \pm 61.2$ & 0.08 \\
\hline LVEF, \% & $26.4 \pm 8.4$ & $27.4 \pm 8.1$ & $27.2 \pm 7.9$ & 0.43 \\
\hline $\mathrm{LA}$ volume index, $\mathrm{mL} / \mathrm{m}^{2}$ & $73.2 \pm 30.2$ & $71.8 \pm 32.0$ & $77.0 \pm 32.3$ & 0.35 \\
\hline
\end{tabular}

Values are presented as numbers (\%) or mean $\pm \mathrm{SD}$, unless otherwise indicated.

BMI, body mass index; BNP, B-type natriuretic peptide; CKD, chronic kidney disease; COPD, chronic obstructive pulmonary disease; CVD, cerebrovascular disease; DM, diabetes mellitus; IHD, ischaemic heart disease; LA, left atrium; LBBB, left bundle branch block; LVEDV, left ventricular end-diastolic volume; LVEF, left ventricular ejection fraction; LVESV, left ventricular end-systolic volume; NYHA, New York Heart Association; Na, serum sodium; RBBB, right bundle branch block; VHD, valvular heart disease; dBP, diastolic blood pressure; sBP, systolic blood pressure.

frequently occurred in the poor adherence group. There was an increased tendency towards 60-day HF rehospitalisation with a decrease in guideline adherence, but the difference was not statistically significant. The incidences of 1-year mortality and the composite end point increased with a decrease in guideline adherence. However, the tendency for 1-year HF rehospitalisation was not different (table 3, figure 2).

\section{Effect of guideline adherence on clinical outcomes}

After adjusting for covariates in the multivariable Cox model, there was a significant trend towards a reduced 60-day mortality ( $\mathrm{p}$ for trend $<0.001$ ) and composite end point $(\mathrm{p}$ for trend $=0.002$ ) with guideline adherence (figure 3). Poor adherence was associated with a significantly higher risk of 60-day mortality compared with moderate (HR 2.74; 95\% CI 1.62 to 4.62 ) and good adherence (HR 4.75; 95\% CI 1.77 to 12.74$)$. It was associated with a higher risk of the 60 -day composite end point compared with moderate (HR 1.61; 95\% CI 1.09 to 2.36 ) and good adherence (HR 2.36; 95\% CI 1.33 to 4.18 ). Furthermore, there was a significant trend towards an increase in 1-year mortality ( $p$ for trend $=0.001$ ) and the composite end point ( $\mathrm{p}$ for trend $=0.02$ ) with guideline adherence. Poor adherence was associated with a significantly higher risk of 1-year mortality compared with moderate (HR 1.64; 95\% CI 1.15 to 2.33) and good adherence (HR 2.34; $95 \%$ CI 1.39 to 3.97 ) and with a higher risk of the 1-year composite end point compared with good adherence (HR $1.58 ; 95 \%$ CI 1.07 to 2.33 ) (figure 3 ). Similar results were 
Table 2 Medical therapy at discharge

\begin{tabular}{|c|c|c|c|c|}
\hline & Poor $(n=147)$ & Moderate $(n=632)$ & Good $(n=207)$ & $P$ value \\
\hline ACEI or ARB & $30(20.4)$ & $535(84.7)$ & $207(100)$ & $<0.001$ \\
\hline ACEI & $12(8.2)$ & $247(39.1)$ & $99(47.8)$ & $<0.001$ \\
\hline Ramipril equivalent dose (mg) & $2.3 \pm 1.4$ & $2.6 \pm 1.8$ & $3.0 \pm 2.2$ & 0.29 \\
\hline ARB & $18(12.2)$ & $251(39.7)$ & $99(47.8)$ & $<0.001$ \\
\hline Candesartan equivalent dose $(\mathrm{mg})$ & $10.2 \pm 7.5$ & $9.8 \pm 6.7$ & $9.9 \pm 6.4$ & 0.75 \\
\hline$\beta$-Blocker & $24(16.3)$ & $355(56.2)$ & $207(100)$ & $<0.001$ \\
\hline Carvedilol equivalent dose (mg) & $12.5 \pm 11.4$ & $19.3 \pm 20.7$ & $16.2 \pm 16.8$ & 0.08 \\
\hline MRA & $129(12.2)$ & $367(58.1)$ & $207(100)$ & $<0.001$ \\
\hline MRA dose at discharge (mg) & $24.0 \pm 13.2$ & $24.0 \pm 13.8$ & $23.4 \pm 14.0$ & 0.90 \\
\hline Warfarin & $38(25.9)$ & $362(57.3)$ & $207(100)$ & $<0.001$ \\
\hline Loop diuretics & $97(66.0)$ & $493(78.0)$ & $180(87.0)$ & $<0.001$ \\
\hline Thiazide diuretics & $10(6.8)$ & $38(6.0)$ & $16(7.7)$ & 0.21 \\
\hline Amiodarone & $21(14.3)$ & $84(13.3)$ & $19(9.2)$ & 0.26 \\
\hline Digitalis & $48(32.7)$ & 347 (54.9) & 127 (61.4) & $<0.001$ \\
\hline
\end{tabular}

Values are presented as numbers (\%) or mean $\pm \mathrm{SD}$, unless otherwise indicated.

ACEI, ACE inhibitor; ARB, angiotensin receptor blocker; MRA, mineralocorticoid receptor antagonists.

observed when mortality was considered a competing risk (online supplementary table).

\section{DISCUSSION}

Our analysis showed that there was a significant trend towards reduced 60-day and 1-year mortality rates and the composite end point with guideline adherence at discharge in patients with acutely decompensated HFrEF with AF. Moreover, poor adherence was associated with a significantly higher risk of 60-day and 1 -year mortality and the composite end point compared with good adherence.

\section{Guideline adherence and outcomes in patients with HF with $\mathrm{AF}$}

The current guideline recommendation is based on clinical trials that demonstrated an improvement in clinical outcomes of HF. Data regarding the efficacy of ACEIs, ARBs or MRAs in decreasing morbidity in patients with HF with concomitant AF, especially admitted patients with AHF, are scarce. Moreover, a recent analysis on the use of $\beta$-blockers in patients with HF with AF documented that $\beta$-blockers did not reduce mortality in patients with HF with AF. ${ }^{14}{ }^{15}$ However, their use is still recommended for reducing adverse remodelling in HF.

Several observational studies have explored the association between class adherence to guideline-recommended therapies and clinical outcomes in HF. ${ }^{8}{ }^{12}$ In the QUALIFY study that recently reported a large international survey for HF (LVEF $\leq 40 \%)$, poor adherence was found to be associated with significantly higher rates of overall mortality, HF hospitalisation, and the composite end point of cardiovascular hospitalisation or cardiovascular death at 6 months. However, the survey included patients with chronic HF and a stable status, and the prevalence of AF was $26.5 \%$. No study has documented the association between guideline adherence and outcomes in patients with $\mathrm{HF}$ and AF. In this study, there was no relationship between HF rehospitalisation and guideline adherence. Although the exact reason is not clear in this study, there are several explanations for the lack of a beneficial effect of guideline-directed therapy in patients with HF with AF. First, the negative inotropic effect of $\beta$-blockers and the potential worsening of haemodynamics can lead to increases in HF rehospitalisation. ${ }^{17}$ The results of the Organised Programme to Initiate Lifesaving Treatment in Hospitalised Patients With Heart Failure registry showed that the use of $\beta$-blockers was associated with a lower risk of rehospitalisation. In that registry, mean LVEF was $39.0 \% .{ }^{18}$ In the Carvedilol Prospective Randomised Cumulative Survival study, which enrolled severe patients with HF with LVED $<25 \%$, the use of $\beta$-blockers was not associated with death or hospitalisation during the first 8 weeks (HR $0.85 ; 95 \%$ CI 0.67 to 1.07 ). ${ }^{19}$ In this study, the mean LVEF was $27 \%$ in the whole patient group. The negative inotropic effect of $\beta$-blockers can be more pronounced in patients with more deteriorated systolic function. Second, worsening renal function (WRF) is an important prognostic factor in patients with HF. In a previous study, initiation of candesartan led to a reduction in renal function, and WRF was associated with worse clinical outcomes, particularly HF hospitalisation. ${ }^{20}$ In another report on KorAHF, the prevalence of WRF in HF was $56.9 \% .{ }^{21}$ Although WRF was not evaluated in this study, it can be suggested that WRF developed more frequently in patients with ACEIs or ARBs, and HF hospitalisation may have increased in the treatment group. These effects

Table 3 Clinical outcomes according to guideline adherence

\begin{tabular}{|c|c|c|c|c|}
\hline & Poor $(n=147)$ & Moderate $(n=632)$ & Good $(n=207)$ & $P$ value \\
\hline Sixty-day heart failure rehospitalisation & 20 (13.6) & $70(11.1)$ & $16(7.7)$ & 0.19 \\
\hline Sixty-day mortality & 27 (18.4) & $40(6.3)$ & $7(3.4)$ & $<0.001$ \\
\hline Sixty-day composite end point & $39(26.5)$ & $104(16.5)$ & $22(10.6)$ & $<0.001$ \\
\hline One-year heart failure rehospitalisation & $31(21.1)$ & $134(21.2)$ & $35(16.9)$ & 0.40 \\
\hline One-year mortality & $45(30.6)$ & $129(20.4)$ & $26(12.6)$ & $<0.001$ \\
\hline One-year composite end point & $62(42.2)$ & $223(35.3)$ & $54(26.1)$ & 0.005 \\
\hline
\end{tabular}

Values are numbers (\%).

Ahn M-S, et al. Heart 2020;106:292-298. doi:10.1136/heartjnl-2019-315240 

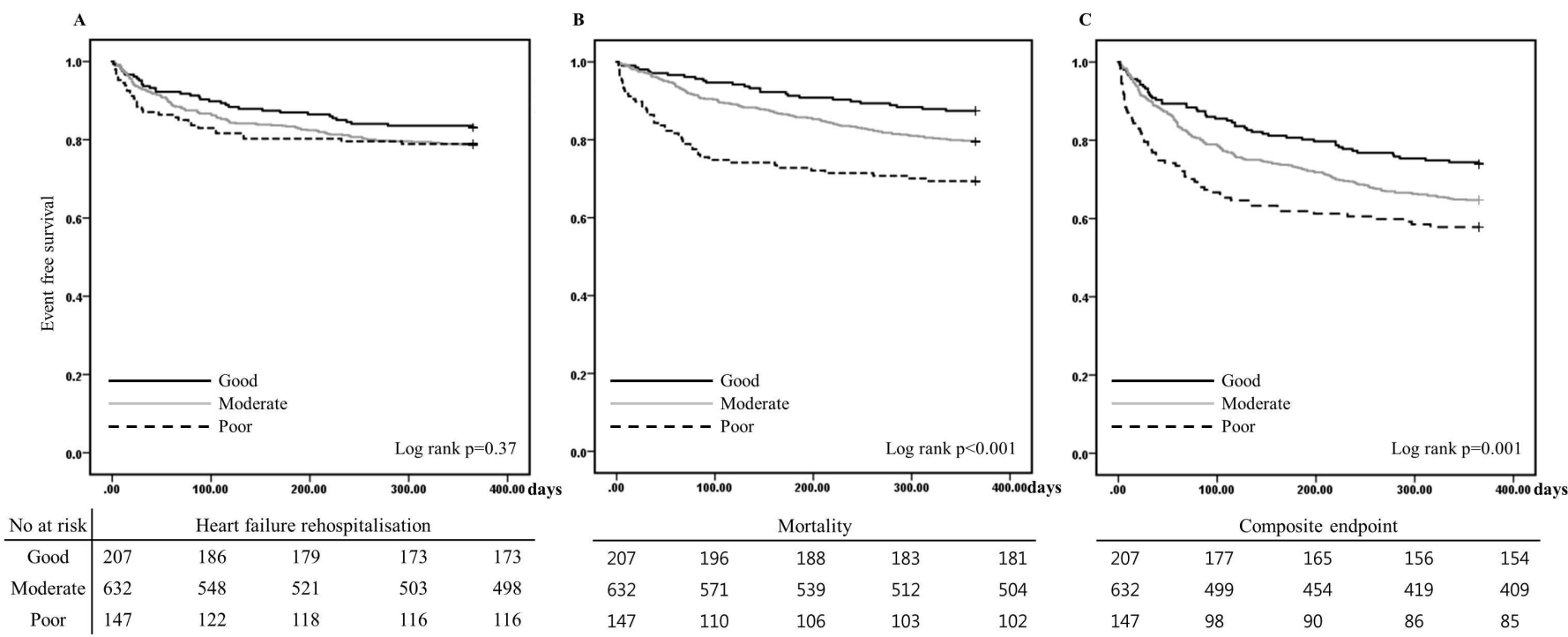

Figure 2 One-year event-free survival according to the guideline adherence score at discharge: (A) heart failure hospitalisation, (B) mortality, (C) composite end point.

can offset the beneficial effect on HF hospitalisation. Third, the beneficial effect of $\beta$-blockers is mediated by reduction of the heart rate, and a lower heart rate is associated with better outcomes in patients with HF with a normal sinus rhythm. However, heart rate does not have the same prognostic significance in patients with $\mathrm{AF}$ as it does in those with a normal sinus rhythm. ${ }^{1422}$ Fourth, AF in patients with HF may be a marker of a poorer clinical condition, leading to a worse outcome that is less modifiable by guideline-directed therapy. ${ }^{23}$
The ACC/AHA/ESC guidelines recommend each therapy unless they are contraindicated or not tolerated. ${ }^{468}$ Several methods have been developed to estimate adherence to clinical guidelines. The performance measures developed by the American College of Cardiology Foundation/AHA/American Medical AssociationPhysician Consortium for Performance Improvement is the ratio of patients prescribed each medication to patients with LV systolic dysfunction and without contraindications for medication. ${ }^{24}$ The patients with contraindications or intolerance were

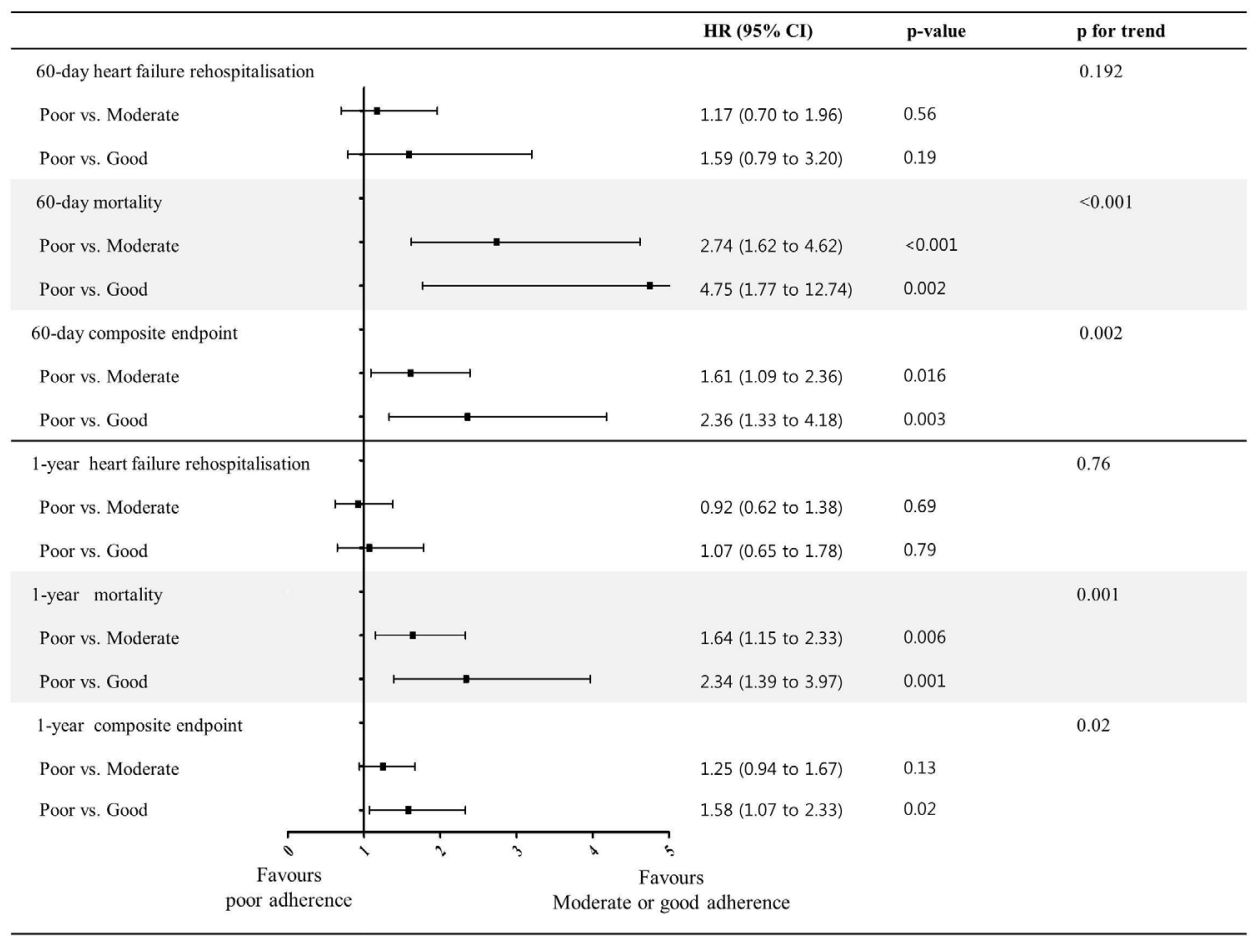

Figure 3 Forest plot of clinical outcomes of poor guideline adherence compared with good and moderate guideline adherence. The HRs and 95\% Cls in comparing the clinical outcomes of heart failure rehospitalisation, mortality and the composite end point, adjusted for sex, age, body mass index, history of hypertension, history of diabetes mellitus, history of ischaemic heart disease, history of chronic obstructive pulmonary disease, type of heart failure (de novo vs acute decompensated heart failure), New York Heart Association functional class, systolic blood pressure, heart rate, creatinine at admission and left ventricular ejection fraction. 
excluded from estimation of guideline adherence. The guidelineadherence indicator indicates the proportion of patients whose physicians prescribed medication according to the guidelines. ${ }^{12}$ Furthermore, non-administration of recommended drugs because of specific contraindications or intolerance was scored as adherence to guidelines. ${ }^{45}$ The modified guideline adherence score in this study strictly assessed whether physicians adhere to guideline recommendations for each individual. As shown in a previous study, our analysis also showed that a poorer guideline adherence was associated with a poorer prognosis in patients with AHF with AF, thus indicating that guideline adherence is important for patients with AHF with AF.

\section{Study limitations}

Several limitations of the present study should be noted. First, this was not a randomised controlled trial but a prospective cohort study. Thus, the HF therapy in the KorAHF registry was not randomly assigned but was entirely dependent on the attending physician, which raises the possibility of selection bias. Furthermore, there may be a risk-treatment mismatch in the guideline-directed therapy. Patients with poor guideline adherence had unfavourable clinical characteristics, but the rates of treatment with guideline-directed therapy were low in highrisk patients. Hence, patients with poor guideline adherence were at a high risk of death. This bias may have influenced the prognostic implications of each therapy despite adjustment for several variables that could influence prognosis. Second, we did not integrate adherence to the target dose of the recommended medication into the adherence score because dose escalation to the target dose is usually performed during the follow-up period after stabilisation. Third, HF therapy may be changed during the follow-up period, and this change may influence the clinical outcomes (online supplementary figure). However, the purpose of this study was to evaluate whether guideline-directed therapy at discharge has prognostic implications. Fourth, AF-specific information was limited, as AF diagnosis was based on electrocardiography at admission or during admission. This may have led to underestimation of AF, as some patients could have had a sinus rhythm during baseline electrocardiography and may have developed (episodes of) AF during follow-up.

\section{CONCLUSION}

Our study showed that a higher level of adherence to guidelines was associated with better 60-day and 1-year prognoses in patients with HF with AF. However, there is a clear unmet need for patients with $\mathrm{HF}$ and $\mathrm{AF}$, given the limited treatment options

\section{Key messages}

What is already known on this subject?

- There are limited and conflicting data regarding the prognostic implications of guideline-directed therapy in patients with heart failure (HF) with reduced ejection fraction and atrial fibrillation (AF).

\section{What might this study add?}

- Better adherence to guidelines is associated with better 60day and 1-year prognoses in patients with HF with AF.

\section{How might this impact on clinical practice?}

- This study suggests that guideline-directed therapy should be started in patients with acute HF with $\mathrm{AF}$, similar to that in patients with a normal sinus rhythm. for this important population. Further investigations are required for the development of optimal therapeutic options for this patient subgroup.

\section{Author affiliations}

'Department of Internal Medicine, Yonsei University, Wonju College of Medicine, Wonju, Republic of Korea

${ }^{2}$ Center of Biomedical Data Science, Yonsei University, Wonju College of Medicine, Wonju, Republic of Korea

${ }^{3}$ Division of Cardiology, Department of Internal Medicine/Cardiovascular Center, Seoul National University Hospital, Jongno-gu, Seoul, Republic of Korea

${ }^{4}$ Department of Internal Medicine and Cardiovascular Center, Seoul National University Hospital, Seoul, Republic of Korea

${ }^{5}$ Division of Cardiology, Sungkyunkwan University College of Medicine, Seoul, Republic of Korea

${ }^{6}$ Division of Cardiology, Severance Cardiovascular Hospital, Yonsei University Wonju

College of Medicine, Seoul, Republic of Korea

${ }^{7}$ Department of Internal Medicine, Seoul National University Bundang Hospital,

Seongnam, Republic of Korea

${ }^{8}$ Chungbuk National University Hospital, Chungju-si, Republic of Korea

Contributors M-SA and BSY had full access to all the data in the study and take responsibility for the integrity of the data and the accuracy of the data analysis. BSY designed the research concept. M-SA wrote the manuscript. All authors contributed to the design and development of the study and to the collection of data. All authors approved the final version of the study.

Funding This work was supported by Research of Korea Centers for Disease Control and Prevention (grant nos. 2010-E63003-00,2011-E63002-00, 2012E63005-00, 2013-E63003-00, 2013-E63003-01, 2013-E63003-02 and 2016ER6303-00)

\section{Competing interests None declared.}

\section{Patient consent for publication Not required.}

Ethics approval The approval for this study was obtained from the ethics committee of each hospital ofKorean Acute Heart Failure registry.

Provenance and peer review Not commissioned; externally peer reviewed. Data availability statement No data are available.

Open access This is an open access article distributed in accordance with the Creative Commons Attribution Non Commercial (CC BY-NC 4.0) license, which permits others to distribute, remix, adapt, build upon this work non-commercially, and license their derivative works on different terms, provided the original work is properly cited, appropriate credit is given, any changes made indicated, and the use is non-commercial. See: http://creativecommons.org/licenses/by-nc/4.0/.

\section{ORCID iDs}

Min-Soo Ahn http://orcid.org/0000-0001-7896-8175 Byung Su Yoo http://orcid.org/0000-0002-3395-4279

Junghan Yoon http://orcid.org/0000-0002-3363-2675

Seung-Hwan Lee http://orcid.org/0000-0002-1186-0917

Jang Young Kim http://orcid.org/0000-0002-0813-7082

Sung Gyun Ahn http://orcid.org/0000-0002-1528-2739

Young Jin Youn http://orcid.org/0000-0001-7066-7474

Jun-Won Lee http://orcid.org/0000-0001-6206-1704

Jung-Woo Son http://orcid.org/0000-0002-1790-3228

Hye Sim Kim http://orcid.org/0000-0002-7431-9722

Dae Ryong Kang http://orcid.org/0000-0002-8792-9730

Hyun-Jai Cho http://orcid.org/0000-0002-2779-4037

Hae-Young Lee http://orcid.org/0000-0002-9521-4102

Eun Seok Jeon http://orcid.org/0000-0002-9946-5611

Seok-Min Kang http://orcid.org/0000-0001-9856-9227

Dong-Ju Choi http://orcid.org/0000-0003-0146-2189

Myeong-Chan Cho http://orcid.org/0000-0002-0047-0227

\section{REFERENCES}

1 Chiang C-E, Naditch-Brûlé L, Murin J, et al. Distribution and risk profile of paroxysmal, persistent, and permanent atrial fibrillation in routine clinical practice: insight from the real-life global survey evaluating patients with atrial fibrillation international registry. Circ Arrhythm Electrophysiol 2012;5:632-9.

2 Wang TJ, Larson MG, Levy D, et al. Temporal relations of atrial fibrillation and congestive heart failure and their joint influence on mortality: the Framingham heart study. Circulation 2003;107:2920-5.

3 Harjola V-P, Follath F, Nieminen MS, et al. Characteristics, outcomes, and predictors of mortality at 3 months and 1 year in patients hospitalized for acute heart failure. Eur $J$ Heart Fail 2010;12:239-48. 
4 Yancy CW, Jessup M, Bozkurt B, et al. 2013 ACCF/AHA guideline for the management of heart failure: a report of the American College of cardiology Foundation/ American heart association Task force on practice guidelines. J Am Coll Cardiol 2013;62:e147-239.

5 Ponikowski P, Voors AA, Anker SD, et al. 2016 ESC Guidelines for the diagnosis and treatment of acute and chronic heart failure: The Task Force for the diagnosis and treatment of acute and chronic heart failure of the European Society of Cardiology (ESC)Developed with the special contribution of the Heart Failure Association (HFA) of the ESC. Eur Heart J 2016:37:2129-200.

6 Yancy CW, Jessup M, Bozkurt B, et al. 2017 ACC/AHA/HFSA focused update of the 2013 ACCF/AHA guideline for the management of heart failure: a report of the American College of Cardiology/American heart association Task force on clinical practice guidelines and the heart failure Society of America. J Am Coll Cardiol 2017;70:776-803.

7 Lee SE, Cho H-J, Lee H-Y, et al. A multicentre cohort study of acute heart failure syndromes in Korea: rationale, design, and interim observations of the Korean acute heart failure (KorAHF) registry. Eur J Heart Fail 2014;16:700-8.

8 Komajda M, Anker SD, Cowie MR, et al. Physicians' adherence to guidelinerecommended medications in heart failure with reduced ejection fraction: data from the QUALIFY global survey. Eur J Heart Fail 2016;18:514-22.

9 Kirchhof P, Benussi S, Kotecha D, et al. [2016 ESC Guidelines for the management of atrial fibrillation developed in collaboration with EACTS]. Kardiol Pol 2016;74:1359-469.

10 January CT, Wann LS, Alpert JS, et al. 2014 AHA/ACC/HRS guideline for the management of patients with atrial fibrillation: a report of the American College of Cardiology/American heart association Task force on practice guidelines and the heart rhythm Society. Circulation 2014;130:e199-267.

11 Corley SD, Epstein AE, DiMarco JP, et al. Relationships between sinus rhythm, treatment, and survival in the atrial fibrillation follow-up investigation of rhythm management (AFFIRM) study. Circulation 2004;109:1509-13.

12 Komajda M, Lapuerta P, Hermans N, et al. Adherence to guidelines is a predictor of outcome in chronic heart failure: the Mahler survey. Eur Heart J 2005;26:1653-9.

13 Austin PC, Fine JP. Practical recommendations for reporting Fine-Gray model analyses for competing risk data. Stat Med 2017;36:4391-400.
14 Kotecha D, Flather MD, Altman DG, et al. Heart Rate and Rhythm and the Benefit of Beta-Blockers in Patients With Heart Failure. J Am Coll Cardiol 2017;69:2885-96.

15 Kotecha D, Holmes J, Krum H, et al. Efficacy of $\beta$ blockers in patients with heart failure plus atrial fibrillation: an individual-patient data meta-analysis. The Lancet 2014;384:2235-43

16 Yoo B-S, Oh J, Hong B-K, et al. Survey of guideline adherence for treatment of systolic heart failure in real world (SUGAR): a multi-center, retrospective, observational study. PLoS One 2014;9:e86596.

17 Jondeau G, Neuder Y, Eicher J-C, et al. B-CONVINCED: beta-blocker continuation vs. interruption in patients with congestive heart failure hospitalizED for a decompensation episode. Eur Heart J 2009;30:2186-92.

18 Hernandez AF, Hammill BG, O'Connor CM, et al. Clinical effectiveness of beta-blockers in heart failure: findings from the OPTIMIZE-HF (organized program to initiate lifesaving treatment in hospitalized patients with heart failure) registry. J Am Coll Cardiol 2009;53:184-92.

19 Krum H, Roecker EB, Mohacsi $\mathrm{P}$, et al. Effects of initiating carvedilol in patients with severe chronic heart failure: results from the COPERNICUS study. JAMA 2003;289:712-8.

20 Damman K, Solomon SD, Pfeffer MA, et al. Worsening renal function and outcome in heart failure patients with reduced and preserved ejection fraction and the impact of angiotensin receptor blocker treatment: data from the CHARM-study programme. Eur $J$ Heart Fail 2016;18:1508-17.

21 Kang J, Park JJ, Cho Young-Jin, et al. Predictors and prognostic value of worsening renal function during admission in HFpEF versus HFrEF: data from the KorAHF (Korean acute heart failure) registry. J Am Heart Assoc 2018;7.

22 Simpson J, Castagno D, Doughty RN, et al. Is heart rate a risk marker in patients with chronic heart failure and concomitant atrial fibrillation? Results from the MAGGIC meta-analysis. Eur J Heart Fail 2015;17:1182-91.

23 Olsson LG, Swedberg K, Ducharme A, et al. Atrial fibrillation and risk of clinical events in chronic heart failure with and without left ventricular systolic dysfunction: results from the candesartan in heart failure-Assessment of reduction in mortality and morbidity (CHARM) program. J Am Coll Cardiol 2006;47:1997-2004.

24 Bonow RO, Ganiats TG, Beam CT, et al. ACCF/AHA/AMA-PCPI 2011 performance measures for adults with heart failure: a report of the American College of cardiology Foundation/American heart association Task force on performance measures and the American medical Association-Physician Consortium for performance improvement. Circulation 2012;125:2382-401. 